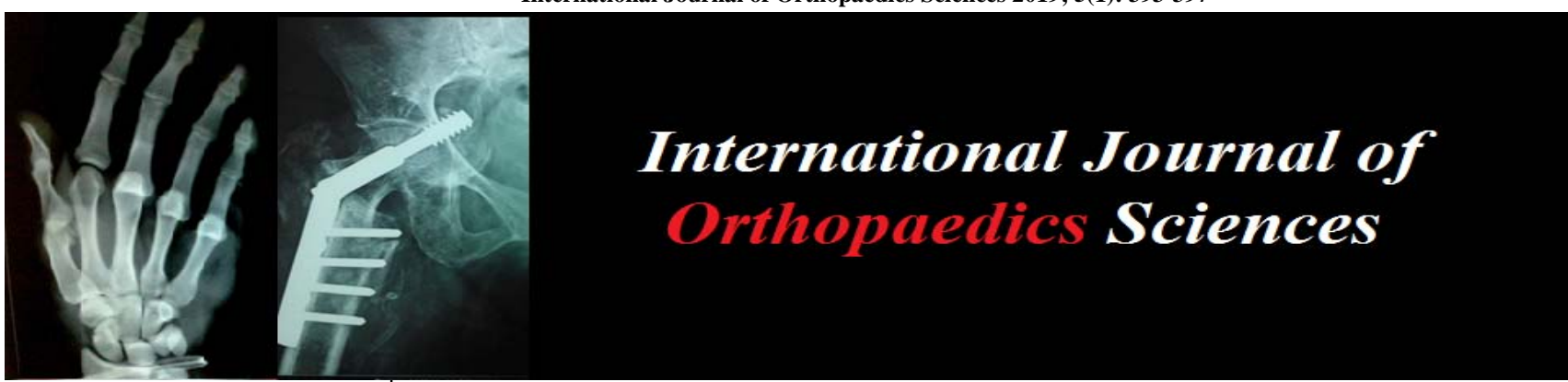

ISSN: $2395-1958$

IJOS 2019; 5(1): 393-397

(C) 2019 IJOS

www.orthopaper.com

Received: 12-01-2019

Accepted: 17-02-2019

Dr. Himanshu Tailor

Assistant Professor,

Department of Orthopaedics,

SMIMER, Surat, Gujarat, India

Dr. Vikram Jasoliya

Assistant Professor,

Department of Orthopaedics,

SMIMER, Surat, Gujarat, India
Correspondence

Dr. Vikram Jasoliya

Assistant Professor,

Department of Orthopaedics,

SMIMER, Surat, Gujarat, India

\section{Results of Posterior and Posterolateral Decompression, Pedicle Screws fixation and Fusion for Dorso-Lumbar Koch's Spine}

\author{
Dr. Himanshu Tailor and Dr. Vikram Jasoliya
}

DOI: https://doi.org/10.22271/ortho.2019.v5.i1g.68

\section{Abstract}

Introduction: Tuberculosis is ubiquitous in distribution. Globally, nearly 30 million people suffer from tuberculosis. Spinal tuberculosis $(50 \%)$ is the most common form of skeletal tuberculosis. In the thoracic and lumbar region, morbidity and complications associated with anterior surgery (Thoracotomy, Retroperitoneal) are very significant. A combined anterior debridement and posterior instrumentation helps to overcome stability related drawbacks of anterior approach alone.

Aims \& Objectives: The aim of this prospective study is to analyse the clinic-radiological outcome of posterior and posterolateral decompression, stabilization with pedicle screws and fusion for tuberculosis of dorsal and lumbar spine done in tertiary care institution from July 2013 to June 2016.

Method:_The Inclusion criteria was age group of 12 to 70 years of age, mild to moderate amount of cold abscess with No improvement with conservative treatment and worsening of neurological deficit. Patients less than 12 years of age, huge cold abscess, severe kyphotic deformity with internal gibbus and patients not fit for anesthesia were excluded from the study.

Results: Before surgery, 9 patients were classified as Frankel grade C, 3 patients each with grade B, D and $\mathrm{E} \& 2$ patient with grade $\mathrm{A}$. After surgery, all patients with grade $\mathrm{C}$ improved to one grade. Out of 3 patients with grade $\mathrm{B}$, one improved to grade $\mathrm{C}$ and other improved to grade $\mathrm{D}$. Of the patients with grade $\mathrm{D}$, one improved to grade $\mathrm{E}$ and the other remained with grade $\mathrm{D}$. One patient with grade $\mathrm{A}$ has not recovered.

One patient developed superficial wound infection which healed by conservative methods and extended antibiotic therapy. Two of our patients developed implant failure. By the time they came for follow up, bony consolidation seen in the follow up x-ray and hence implant exit done.

Conclusion: The posterior/posterolateral approach (extracavitary approach) gives a reasonable access to the lateral and anterior aspects of the cord for an equally good decompression of the cord, avoids problems of prolonged recumbency, provides better functional outcome and significantly better kyphosis correction.

Keywords: Tuberculosis, Koch's spine, Posterolateral decompression, Pedicle screw fixation

\section{Introduction}

Tuberculosis is ubiquitous in distribution. Globally, nearly 30 million people suffer from tuberculosis. 3 million deaths occur due to tuberculosis per year. India has burden of 6 million cases. Recent increase in the incidence is due to concomitant tuberculosis with HIV infection and drug resistance. Of these $1-3 \%$ constitutes skeletal system involvement. Spinal tuberculosis $(50 \%)$ is the most common form of skeletal tuberculosis ${ }^{[1]}$ The evolution of treatment of tuberculosis of spine have passed through different phases of development from Pre-antitubercular era through Post antitubercular era and from Radical surgery through Middle path regimn ${ }^{[3]}$ Traditionally, the anterior approach is the gold standard approach because vertebral bodies and disc spaces are most commonly affected, and the anterior approach allows direct access to the diseased vertebral bodies for debridement and abscess drainage, allows wide decompression and reconstruction of the defect. ${ }^{[1,5]}$ In the thoracic and lumbar region, morbidity and complications associated with anterior surgery (Thoracotomy, Retroperitoneal) are very significant. Anterior instrumentation to provide bone stability may be tenuous because the concomitant osteoporosis associated with infection renders the vertebrae structurally weak, prevent adequate fixation and the construct is biomechanically 
less stable ${ }^{[3,7]}$. The approach to the upper thoracic spine is difficult and there is a need for thoracic surgeon. The lung may be scarred or adherent to the underlying pleura which precludes the anterior approach.

A combined anterior debridement and posterior instrumentation helps to overcome stability related drawbacks of anterior approach alone [6,7]. However, it entails two surgeries (single event or staged) with additional morbidity and is indicated for patients with significant deformity. In posterior or posterolateral approaches anterior and lateral column can be reached through extra pleural approach. Posterior approach provides excellent exposure for circumferential spinal cord decompression, allows multiple level posterior instrumentation above and below the level of pathology, more stable construct, less morbid surgery, allows earlier rehabilitation and is a familiar approach ${ }^{[1,7]}$. In this study we clinic-radiologically evaluated the outcome of posterior and posterolateral decompression, stabilization with pedicle screws and fusion for tuberculosis of dorsal and lumbar spine.

\section{Aims and Objectives}

The aim of this prospective study is to analyse the clinicradiological outcome of posterior and posterolateral decompression, stabilization with pedicle screws and fusion for tuberculosis of dorsal and lumbar spine done in tertiary care institution from July 2013 to June 2016.

\section{Materials and Methods}

This study was conducted in our Hospital on 20 patients with Tuberculosis of Dorsal and Lumbar spine from July 2013 to June 2016. All patients were treated with posterior or posterolateral approach and stabilized with Pedicle screw system.

\section{Objectives}

a) To study the effectiveness of posterior and posterolateral decompression, stabilization with pedicle screws and fusion for tuberculosis of dorsal and lumbar spine.

b) To study the improvement in the angle of kyphosis.

c) To show that posterior surgery allows early mobilization.

d) To evaluate that posterior surgery associated with reduced morbidity and mortality.

\section{Selection criteria}

The Inclusion criteria was age group of 12 to 70 years of age, mild to moderate amount of cold abscess with No improvement with conservative treatment and worsening of neurological deficit.

Patients less than 12 years of age, huge cold abscess, severe kyphotic deformity with internal gibbus and patients not fit for anesthesia were excluded from the study.

\begin{tabular}{|c|c|}
\hline Age of Involvement & No. of Patients \\
\hline $10-20$ & 3 \\
\hline $21-30$ & 5 \\
\hline $31-40$ & 7 \\
\hline $41-50$ & 3 \\
\hline $51-60$ & 1 \\
\hline $61-70$ & 1 \\
\hline
\end{tabular}

\begin{tabular}{|c|c|}
\hline Sex & No. of Patients \\
\hline Male & 11 \\
\hline Female & 9 \\
\hline
\end{tabular}

\begin{tabular}{|c|c|}
\hline Mode of Presentation & No. of Patients \\
\hline Pain & 13 \\
\hline Paraparesis & 6 \\
\hline Deformity & 1 \\
\hline Level of Lesion & No. of Patients \\
\hline D1-D10 & 6 \\
\hline D11-L2 & 9 \\
\hline L3-L5 & 5 \\
\hline
\end{tabular}

During study age of patient, mode of presentation, Level of the lesion and associated co morbid condition are considered. Anti tuberculous therapy started.

\section{Pre-operative planning}

Pain evaluated by Visual analog score. Neurological status documented by Frankel's grade. Radiographic examination includes AP view, Lateral view of the dorsal or lumbar spine, CT scan and MRI.

\section{Per operative evaluation}

During surgery operative blood loss, operative times were noted.

Anesthesia: General anesthesia.

\section{Position of the Patient}

Prone with Bolsters are placed longitudinally under the patient's sides to allow the abdomen to be entirely free. Pressure points are carefully padded. After preparing and draping the surgical site, Skin and subcutaneous tissue infiltrated with Tumescent solution.

\section{Incision}

Midline longitudinal incision made over the spinous processes, extending from the spinous process above to the spinous process below the pathologic level. The length of the incision depends on the number of levels to be explored.

\section{Post-op protocol}

1. Early mobilization from the bed with hyperextension brace

2. Muscle strengthening exercises as soon as the pain subsides

3. Forward and backward bending after radiological healing

4. Range from 8 months to 2 years

\section{Post-op follow up}

The patients are followed up at 3 months, 6 months, 1 year and every 6 months and evaluated for the Visual analogue pain scale and the functional outcome evaluated using Postoperative Frankel grading, Angle of kyphosis, Oswestry disability index.

\section{Results}

This study was conducted in our Hospital on 20 patients with Tuberculosis of Dorsal and Lumbar spine from July 2013 to June 2016. All patients were treated with posterior or posterolateral approach and stabilized with Pedicle screw system. The mean follow up period was 12 months (range 626 months). 
The following observations are made in this study

There was almost equal distribution in male \& female. The most common age group affected was $3^{\text {rd }}$ and $4^{\text {th }}$ decade $(60 \%)$. The most common mode of presentation is pain (66.7\%). The dorsolumbar junction is most commonly affected (46.7\%).

\section{The following are the results of the study}

The mean duration between surgery and onset of symptoms was 10.2 months (range 5-14 months). The mean surgical time was 3 hours 20 minutes (range $2 \mathrm{~h} 20 \mathrm{~min}-4 \mathrm{hr} 10 \mathrm{~min}$ ). The average blood loss was $800 \mathrm{ml}(400 \mathrm{ml}-1500 \mathrm{ml})$. The mean preoperative Visual analog score was 8.7 (range7-10) which improved to 1.7(1-4) at final follow up implying better pain score postoperatively. The mean preoperative ESR value was 111.8 which improved to 31.7 at final follow up which indicates improvement in disease activity. Before surgery, 9 patients were classified as Frankel grade C, 3 patients each with grade $\mathrm{B}, \mathrm{D}$ and $\mathrm{E} \& 2$ patient with grade A. After surgery, all patients with grade $\mathrm{C}$ improved to one grade. Out of 3 patients with grade $B$, one improved to grade $C$ and other improved to grade D. Of the patients with grade D, one improved to grade $\mathrm{E}$ and the other remained with grade $\mathrm{D}$. One patient with grade A has not recovered.

\begin{tabular}{|c|c|}
\hline \multicolumn{2}{|c|}{ Mean VAS Score } \\
\hline Pre-op & 8.7 \\
\hline Post-op & 2.5 \\
\hline
\end{tabular}

\begin{tabular}{|l|l|}
\hline Mean ESR \\
\hline Pre-op & 111.8 \\
\hline Post-op & 2.5 \\
\hline
\end{tabular}

\begin{tabular}{|c|c|}
\hline \multicolumn{2}{|c|}{ Mean Kyphotic Angle } \\
\hline Pre-op & 27.9 \\
\hline Post-op & 9.5 \\
\hline
\end{tabular}

The mean preoperative kyphosis in the thoracic and thoracolumbar spine was 27.9 degrees which was corrected to a mean of 9.5 degrees in the final follow up radiographs implying better correction and maintenance of kyphosis.

\section{Complications}

One patient developed superficial wound infection which healed by conservative methods and extended antibiotic therapy. Two of our patients developed implant failure. By the time they came for follow up, bony consolidation seen in the follow up x-ray and hence implant exit done.

\section{Discussion}

The treatment of tuberculosis of spine consists of conservative methods or surgical management. Conservative method comprises Bed rest with or without Plaster casts, Chemotherapy, Supervision with Imaging and blood markers every 3 months followed by resumption of activity with braces. It requires long period of immobilization and it leads to complications of prolonged recumbency like deep vein thrombosis, bed sore and chest infection. It cannot prevent the progression of kyphotic deformity ${ }^{[2,17]}$ To circumvent the problems associated with conservative management and those who did not show signs of progressive recovery, development of neurological problems, neurological worsening during conservative therapy, advanced cases and in the elderly, surgery is indicated. The goals of surgery in Tuberculosis of Thoracic and Lumbar spine are adequate decompression, adequate debridement, maintenance and reinforcement of stability and correction and to stop the progression of Kyphosis ${ }^{[21]}$.

Among the various types of decompression, anterior approach is considered the gold standard for debridement and decompression in Pott's spine. Anterior radical surgery was popularized by Hodgson and Stock in $1960{ }^{[4]}$. Advantages of the traditional anterior approach are ability to directly access the disease and perform decompression, better correction of deformity, less muscle dissection and the ability to place a graft under compressive load for fusion. The disadvantages of anterior approach are morbidity and mortality associated with the transpleural and retroperitoneal approach like atelectasis, chest infection, pneumothorax and postoperative ileus, increase in spinal instability after surgical decompression in the immediate postoperative period. Also when the patient has coexisting pulmonary pathology, the approach may be difficult ${ }^{[24]}$. The structural bone graft in anterior approach does not give initial stability and graft related problems occur more often when the graft spans more than two-disc space. The tricortical iliac crest graft is associated with donar site morbidity. The rib grafts are readily available after thoracotomy but they are structurally weak and do not contain cancellous bone ${ }^{[12]}$.

The stability of anterior instrumentation may not provide adequate fixation as there is concomitant inflammation associated with infection and the anterior bones are hyperemic and porotic. Implant holding is a problem and there might be risk of graft subsidence and graft slippage. It was during 1970's that the modern spinal instrumentation was developed and came to use. There was an initial apprehension to use the metal implants in active infection. Oga et al. ${ }^{[23]}$ studied the adherence capacity of Mycobacterium tuberculosis to stainless steel and concluded that adherence was negligible and the use of implants in regions with active tuberculosis infection may be safe and the tubercle bacilli, unlike pyogenic organisms do not adhere to metal and form any biofilm. Posterior instrumentation with anterior decompression and fusion can be performed in one or two stages ${ }^{[26]}$. This surgery is more radical but recurrence rate is low. However, if performed in one stage, the procedure has more morbidity. When anterior decompression and bone grafting is performed as a first stage procedure, there is a risk of graft slippage and neural deterioration while waiting for second stage stabilization. In the second stage, only in situ stabilization will be performed. When the posterior procedure is performed first, it will be only in situ stabilization followed by secondstage decompression, so kyphus correction will be minimal. Guven and coworkers ${ }^{[25]}$ after using posterior instrumentation in the form of pedicle screw or hook system for thoracolumbar tuberculosis of the spine

have found clinical and radiological evidence of stable fusion in all their patients.

In this study, we used transpedicular screws with Moss Miami system because it has been proven to be a good method for stabilizing the thoracolumbar and lumbar spine. It is not radical as that of the anterior approach. During instrumentation of the pedicle screws in thoracic and the lumbar spine, we inserted the screws into the healthy pedicles adjacent to the areas of bone destruction. There is a quick relief of instability ${ }^{[28]}$ in patients treated with early stage spinal tuberculosis with transpedicular instrumentation. This posterior approach has a special advantage in that it avoids contact with the infectious focus, because the tuberculous infection usually involves the anterior column. The fact that 
tuberculous lesions may heal in spontaneous fusion makes this posterior approach feasible. The transpedicular instrumentation helps in maintaining the spinal alignment and stabilization of the involved vertebra. The posterior instrumentation acts as a spacer and allows the healing of the anterior mass and supports the anterior column ${ }^{[29]}$ Posterior instrumentation has been reported to be quite effective in preventing graft related complications and progression of kyphosis. The main advantage of posterior instrumentation is that it can provide good fixation through posterior elements as the disease pathology is anterior. Posterior fixation also helps in correcting pre-existing kyphosis effectively. Posterior approach utilizing only extra pleural approach, as described by Jain et al., ${ }^{[1]}$ is an effective option. Extra pleural approach allows decompression of spinal cord under direct vision and supplemented with a stable posterior instrumentation, which has the multilevel flexibility to be extended above and below if needed.

The cord was decompressed by excising a pedicle in order to allow access to the anterior debris. A limited debridement was undertaken to decompress the cord without creating a large defect. The exposure for the decompression may be improved by sacrificing a spinal nerve root at the level of the excised pedicle in the thoracic spine. This does not significantly affect the morbidity because of the overlap of the dermatomes and circumvents the problems related to a thoracotomy. The transpedicular route for decompression is preferable to the transthoracic procedure since it allows an adequate global removal of the anterior, lateral and posterior cuff of tissue, posterior stabilisation is possible through the same approach and chest complications related to thoracotomy in these patients are avoided. The posterior instrumentation allows early mobilisation, thus avoiding the complications of prolonged recumbency. The stability provided by posterior fixation, particularly transpedicular fixation, protects the vertebral correction, and patients are able to return to normal activities within a short period of time ${ }^{[28]}$ Poor sagittal spinal correction has been documented following anterior approach alone. While anterior instrumentation may prevent progression of kyphosis during treatment, it is not so effective in correcting pre-existing kyphosis. Addition of posterior instrumentation has shown to improve correction of sagittal alignment. Reported kyphosis correction ranges from initial $30^{\circ}-35^{\circ}$ to $15^{\circ}-18^{\circ}$ postoperatively, with $2^{\circ}-3^{\circ}$ loss of correction with an average followup of 45 months. ${ }^{1}$ In our case series also, the kyphosis correction was significantly better with posterior approach alone.

Bhavuk Garg et al. in a retrospective analysis of 70 patients with thoracic and lumbar tuberculosis compared the clinical, radiological and functional outcome of anterior versus posterior debridement and stabilization concluded that though the anterior approach is an equally good method for debridement and stabilization, kyphus correction is better with posterior instrumentation and the posterior approach is associated with less morbidity and complications. GD Sunderraj et al. ${ }^{[28]}$ in a prospective study of patients with tuberculosis of dorsal, dorsolumbar and lumbar spine combined with anterior or posterior instrumentation surgery concluded that adjuvant posterior stabilization allows early mobilization and rehabilitation. Graft related problems were fewer and the progression and the maintenance of correction of the kyphosis were better than with anterior surgery alone. Even when large amount of pus present there is no implant related risk. Moon et al. ${ }^{8}$ retrospectively analysed 124 patients with spinal tuberculosis in children and concluded that posterior instrumented stabilization alone could correct and prevent progress of kyphosis. Lee et al. ${ }^{[7]}$ in a retrospective case series using single stage Transpedicular decompression and posterior instrumentation of thoracic and thoracolumbar tuberculosis concluded that it can be an alternative treatment method of less involved spinal tuberculosis especially for patients in early phase of bone destruction or ones with mild kyphosis. We prefer a posterior approach because familiarity of the approach and the complication rate is low. The benefits of our approach are early ambulation, decreased morbidity, and good access to dural abscesses, sequestrated bone and small abscesses in the anterior spine. Posterior instrumentation can be used to correct deformity and any concurrent spinal stenosis in elderly patients can be treated simultaneously. The results of our study show that thoracic and lumbar tuberculous spondylitis can be successfully treated through the posterior approach by meticulous debridement of necrotic bone and the infected disc. It allows easy access to the spinal canal for neural decompression, prevents loss of correction of vertebral alignment, and facilitates early mobilisation.

\section{Conclusion}

The posterior/posterolateral approach (extracavitary approach) gives a reasonable access to the lateral and anterior aspects of the cord for an equally good decompression of the cord. It is a less morbid approach and avoids problems associated with thoracotomy and laparotomy.

It facilitates early mobilization and avoids problems of prolonged recumbency. It provides better functional outcome and significantly better sagittal plane and kyphosis correction. Posterior approach preferred because of its familiarity, its simplicity, and its low complication rate.

\section{References}

1. Jain AK, Dhammi IK, Prashad B. Simaltaneous anterior decompression and posterior instrumentation of the tuberculosis spine using an anterolateral extrapleural approach. J Bone Joint Br. 2008; 90:P1477-81.

2. Tuli SM. Tuberculosis of the skeletal system.4th edition, 19:193-194.

3. Hee HT, Majd ME, Holt RT. Better treatment of vertebral osteomyelitis using posterior stabilization and titanium mesh cages. J Sinal Disord Tech. 2002; 15:14956.

4. Hodgson AR, Stock FE, Fang HS, Ong GB. Anterior spinal fusion: The operative approach and the pathological findings in 412 patients with Pottes disease of the spine. Br J Surg. 1960; 48:172-8.

5. Fukuta S, Miyamato $\mathrm{K}$, Masuda $\mathrm{T}$ et al. Two stage surgical treatment using posterior spinal instrumentation for pyogenic and tuberculous spondylitis. Spine. 2003; 28:E302-8.

6. Krodel A, Kruger A, Pfahler M, Anterior debridement, fusion and extrafocal stabilization in the treatment of osteomyelitis of spine.J spinal Disord. 1999; 8:304-9.

7. Lee $\mathrm{TC}, \mathrm{Lu} \mathrm{K}$, Yang LC, Huang HY, Liang CL. Transpedicular instrumentation as an adjunct in the treatment of thoracolumbar and lumbar spine tuberculosis with early state bone destruction. J Neurosurg. 1999; 91(2 Suppl):163-9

8. Moon MS, Woo YK, Lee KS et al. Posterior instrumentation and anterior interbody fusion for tuberculous kyphosis of dorsal and lumbar spine. Spine. 1995; 20:1910-16. 
9. Tuli SM. Tuberculosis of the skeletal system.3rd edition; 2003, 193-7.

10. Gray"s Anatomy. The Anatomical basis of Clinical practice: 39 th edition, 45 .

11. Rajasekaran S, Anil K Jain, Ajoy P Shetty. Spinal infections and trauma. $1^{\text {st }}$ edition, 2011, 66-68.

12. Rajasekaran S, Shanmugasundaram TK. Prediction of the angle of gibbus deformity in tuberculosis of the spine. $\mathrm{J}$ Bone Joint Surg [Am] 1987; 69-A:503-9.

13. Ananthanarayanan, Panikar. Textbook of microbiology. 7 th edition, 39.

14. Donowitz GR, Mandell GL. Acute pneumonia. In: Mandell GL, Bennet JE, Dolin R. Principles and practice of Infectious diseases, $6^{\text {th }}$ edn.Philadelphia. 2005, 819-45.

15. Rajasekaran S. The natural history of post-tubercular kyphosis in children: radiological signs which predict late increase in deformity. J Bone Joint Surg [Br]. 2001; 83B:954-62.

16. Thijn CJ, Steensma JT. Tuberculosis of the skeleton: Focus on radiology. New York: Springer Verlag, 1990.

17. Tuli SM. Tuberculosis of the skeletal system.4th edition. 19:193-194.

18. Tuli SM. Tuberculosis of the skeletal system.4th edition. 22(22.3):242.

19. Hadjipavlou AG, Kontakis GM, Gaitanis J Net al.,Effectiveness and pitfalls of percutaneous transpedicle biopsy of spine.Clin Orthop Relat Res. 2003; 411:54-60.

20. Lin YS, Haung YC, Chang LY. Clinical characteristics of tuberculosis in children in the north of Taiwan.J Microbial Immunol Infect. 2005; 38:41-6.

21. Benli T, Kaya A, Acaroglu E. Anterior instrumentation in tuberculous spondylitis: Is it effective and safe? Clin Orthop Relat Res. 2007; 460:108-16.

22. Pandit HG, Sonsale PD, Shikare SS. Bone scintigraphy in tuberculous spondylodiscitis. Eur Spine J. 1998; 8:205-9.

23. Oga M, Arizono T, Takasita $\mathrm{M}$ et al: Evaluation of the risk of instrumentation as a foreign body in spinal tuberculosis. Clinical and biologic study. Spine. 1993; 18:1890-1894,

24. Hodgson AR, Yau ACMC, Kwon JS. A clinical study of 100 consecutive cases of Pott's paraplegia. Clin Orthop 1964; 36:128-150,

25. Güven O, Kumano K, Yalçin S, Karaham M, Tsuji S. A single stage posterior approach and rigid fixation for preventing kyphosis in the treatment of spinal tuberculosis. Spine. 1994; 19:1039-43.

26. Jin D, Qu D, Chen J, Zhang H. One-stage anterior interbody autografting and instrumentation in primary surgical management of thoracolumbar spinal tuberculosis. Eur Spine J. 2004; 13:114-21.

27. Chen WJ, Chen $\mathrm{CH}$, Shih $\mathrm{CH}$. Surgical treatment of tuberculous spondylitis: 50 patients followed for 2-8 years. Acta Orthop Scand. 1995; 66:137-42.

28. Sundararaj GD, Behera S, Ravi V et al. Role of posterior stabilisation in the management of tuberculosis of the dorsal and lumbar spine. J Bone Joint Surg [Br]. 2003; 85-B:100-6.

29. Lee TC, Lu K, Yang LC, Huang HY, Liang CL. Transpedicular instrumentation as an adjunct in the treatment of thoracolumbar and lumbar spine tuberculosis with early state bone destruction. J Neurosurg. 1999; 91(2 Suppl):163-9. 